\title{
Assessment of relationships among clinicopathological characteristics, morphological computer tomography features, and tumor cell proliferation in stage I lung adenocarcinoma
}

\author{
Xiaoling Ma, Shuchang Zhou, Lu Huang, Peijun Zhao, Yujin Wang, Qiongjie Hu, Liming Xia \\ Department of Radiology, Tongji Hospital, Tongji Medical College, Huazhong University of Science and Technology, Wuhan, China \\ Contributions: (I) Conception and design: X Ma; (II) Administrative support: L Xia; (III) Provision of study materials or patients: X Ma, S Zhou, L \\ Huang, P Zhao, Y Wang, Q Hu; (IV) Collection and assembly of data: X Ma, S Zhou, L Huang, P Zhao, Y Wang, Q Hu; (V) Data analysis and \\ interpretation: X Ma; (VI) Manuscript writing: All authors; (VII) Final approval of manuscript: All authors. \\ Correspondence to: Liming Xia. Department of Radiology, Tongji Hospital, Tongji Medical College, Huazhong University of Science and Technology, \\ 1095 Jiefang Road, Qiaokou District, Wuhan 430030, China. Email: xialiming2017@outlook.com.
}

\begin{abstract}
Background: Surgically resected stage I lung adenocarcinoma (ADC) has wide variation in prognosis. It is significant to identify high-risk patients and optimize therapeutic strategy. This study aimed to investigate the relationships among histological grade, serum tumor marker index (TMI), morphological computer tomography (CT) features, and a well-established prognosticator cell proliferation (Ki-67) in stage I ADC.

Methods: Preoperative CT was performed in 182 patients with stage I ADC confirmed by pathology. The Ki-67 expression was acquired by immunohistochemistry. TMI was the square root of standardized serum carcinoembryonic antigen (CEA) and cytokeratin 19 fragments (CYFRA 21-1) values. Tumor shadow disappearance rate (TDR) and other morphological CT features were interpreted by two radiologists. Histological grade, TMI, CT features were statistically evaluated to explore the associations with Ki-67 expression.
\end{abstract}

Results: In univariate analysis, gender, smoking history, pack-year, histological grade, TNM stage (IA and IB), serum CEA and CYFRA 21-1 status, TMI status, as well as TDR, long-axis diameter, short-axis diameter, lobulation, spiculation, attenuation types, vacuolation, vascular invasion, vascular convergence, thickened bronchovascular bundles, pleural attachment and peripheral fibrosis were significantly associated with Ki-67 expression (all $\mathrm{P}<0.05$ ). Solid-predominant ADC had the highest Ki-67 expression, followed by micropapillary, papillary and acinar-predominant ADC, while lepidic-predominant ADC had the lowest Ki-67 expression $(\mathrm{P}<0.001)$. TDR was negatively correlated with Ki-67 ( $\mathrm{r}=-0.478, \mathrm{P}<0.001)$. Multivariate logistic regression analysis revealed that gender, histological grade, TDR and attenuation types were independent factors associated with Ki-67 expression.

Conclusions: Ki-67 expression differed distinctly according to ADC histological subtypes. High Ki-67 expression is independently associated with male patients of stage I ADC with worse differentiation, lower TDR and solid tumors, which might be of prognostic value for poor prognosis in stage I ADC.

Keywords: Lung adenocarcinoma; stage I; Ki-67; computer tomography; serum tumor marker

Submitted Jan 01, 2021. Accepted for publication Mar 25, 2021.

doi: $10.21037 /$ jtd-21-7

View this article at: http://dx.doi.org/10.21037/jtd-21-7

\section{Introduction}

Non-small cell lung cancer (NSCLC) accounting for $80 \%$ of lung cancer is one of the most common causes for cancer- related death and poses a tremendous threat to human health (1). Even in stage I NSCLC, as typically prescribed with a radical surgical resection, $30-60 \%$ of patients would 
suffer from postsurgical recurrence, lowering the chance of 5-year survival (2). To date, though no consensus on postsurgical chemotherapy for stage I NSCLC has yet been reached, there is growing evidence approving the positive effect of adjuvant chemotherapy in high-risk early-stage NSCLC $(3,4)$. As the main histological subtype of NSCLC, lung adenocarcinoma (ADC) show different characteristics from other subtypes in profiles of molecular genotyping and biological behaviors. It is of great significance to identify early-stage ADC patients with poor prognosis, who will be candidates for adjuvant chemotherapy besides routine surgical resection. The International Association for the Study of Lung Cancer (IASLC), American Thoracic Society (ATS), and European Respiratory Society (ERS) have proposed a classification system for ADC in 2011 to classify invasive adenocarcinoma into different subtypes according to the predominant histological pattern (5). Recently, a new grading system for invasive ADC was endorsed by the IASLC pathology committee, which took the proportion of high-grade patterns into consideration and revealed a superior performance in prognostic classification (6).

$\mathrm{Ki}-67$ is a well-characterized proliferation-associated nuclear antigen, exclusively existing during active phases of the cell cycle (i.e., G1, S, G2 and mitosis), and is widely considered as an indicator for prognosis in various types of carcinoma $(7,8)$. A recent meta-analysis including 108 studies based on 14,831 patients highlighted the prognostic value of Ki-67 expression for lung cancer (9). Furthermore, some previous studies demonstrated that high Ki-67 labeling index (LI) had an adverse prognostic impact on ADC (10-12). Preoperative serum carcinoembryonic antigen (CEA) and cytokeratin 19 fragments (CYFRA 21-1) were established indicators of pathological tumor invasion. The elevated serum CEA and CYFRA were closely associated with poor prognosis in NSCLC $(13,14)$. Additionally, we introduced a synthetical metric named tumor marker index (TMI), which is derived from serum CEA and CYFRA 21-1 values. TMI was previously used to identify patients at high risk of relapse and was a strong prognostic marker for earlystage NSCLC reported by Muley et al. (15). With the wide application of computed tomography (CT), it serves as a useful tool to screen tumors, monitor therapy response, and perform regular follow-up. Morphological features on CT by visual inspection were developed as potential biomarkers to predict genetic mutation, distinguish pre-invasive and invasive lesions, and prognosticate outcomes in lung cancer (16-18).

Until now, although Ki-67, histological grade, TMI and morphological CT features were potential prognostic factors for survival in lung cancer, the relationships among histological grade, TMI, CT features, and Ki-67 in stage I ADC have not been previously reported. This study aimed to investigate whether histological grade, morphological CT features and TMI are significantly associated with Ki67 expression in stage I ADC.

We present the following article in accordance with the MDAR checklist (available at http://dx.doi.org/10.21037/ jtd-21-7).

\section{Methods}

\section{Patient}

The study was conducted in accordance with the Declaration of Helsinki (as revised in 2013). This study was approved by the Institutional Review Board (NO. TJIRB-20180615). Individual consent for this retrospective analysis was waived.

From April 2018 to January 2019, patients who were hospitalized in the thoracic surgery department of our hospital were retrospectively reviewed. The inclusion criteria were as followed: (I) all patients underwent surgery with thoracoscopic lobectomy, lobectomy, wedgeshaped resection, segmental lung resection or others, and systematic lymph node excision; (II) patients with primary stage I ADC were diagnosed by postoperative pathology; (III) the acquisition of preoperative CT within two weeks before surgery was available; and (IV) valid test report of Ki-67 LI through immunohistochemistry. Meanwhile, patients were excluded by the following criteria: (I) patients with adenocarcinoma in situ and minimally invasive adenocarcinoma, who have excellent prognoses after radical surgical resection; (II) patients subjected to other cancers; (III) preoperative radiotherapy or chemotherapy; (IV) CT images with obvious artifacts. Clinicopathological characteristics including age, gender, smoking history, packyear, histological grade, Tumor-Node-Metastasis (TNM) stage, lobe location, and preoperative serum CEA, CYFRA 21-1 and TMI were recorded.

\section{Histological evaluation and grading}

Invasive ADC was classified into five subtypes of lepidic (LPA), acinar (APA), papillary (PPA), micropapillary (MPA) and solid predominant adenocarcinoma (SPA) based on histological patterns (5). According to the new grading 
system endorsed by the IASLC pathology committee in 2020 (6), invasive ADC was categorized into three histological grades: well-differentiated (LPA with no or less than $20 \%$ of high-grade patterns); moderatelydifferentiated (APA or PPA with no or less than $20 \%$ of high-grade patterns); and poorly-differentiated ADC (any tumor with $20 \%$ or more of high-grade patterns). The high-grade patterns included solid, micropapillary, and complex glandular patterns.

\section{CT imaging acquisition}

Preoperative CT examinations were routinely underwent using one of these equipment (GE Discovery CT750 HD, or GE Optima 660, GE Healthcare) with bone reconstruction algorithm. The CT acquisition parameters were typically: tube voltage, $120 \mathrm{kV}$; tube current, $250-300 \mathrm{~mA}$; pitch, $0.98-1.00 \mathrm{~mm}$; field of view, $350 \times 350 \mathrm{~mm}$; matrix, $512 \times 512$; slice thickness, $5 \mathrm{~mm}$; reconstruction thickness and thickness interval, 1.25 and $1.25 \mathrm{~mm}$; contrast agent (Ultravist, Iopromide, Bayer Schering Pharma AG, Leverkusen, Germany) bolus at the speed of $2.5-3.5 \mathrm{~mL} / \mathrm{s}$ was injected into the right elbow vein with $1.1 \mathrm{~mL} / \mathrm{kg}$ of weight; scan delay time was $30 \mathrm{~s}$ and breathhold was requisite.

\section{CT imaging review}

All CT characteristics were interpreted independently by two chest radiologists (ZSC and MXL) with imagingdiagnostic experience of 13 years and 5 years respectively, who were blinded to patients' clinicopathological information and Ki-67 expression level. For continuous variables, the final values were determined using averages. For categorical variables, a consensus was reached with the third radiologists (HQJ with 11 years of experience) joined when a divergence occurred. Lung window (width, 1,600 $\mathrm{HU}$; level, $-600 \mathrm{HU}$ ) and mediastinal window (width, $400 \mathrm{HU}$, level, $40 \mathrm{HU}$ ) was fixed to review CT images. Definitions of CT features were listed in Table 1. Tumor shadow disappearance rate (TDR) was calculated with the following formula: $\operatorname{TDR}(\%)=\left(1-\mathrm{MaxD}_{\text {mediastinal }} \times\right.$ $\left.\max D_{\text {mediastinal }} / \mathrm{MaxD}_{\text {lung }} \times \max \mathrm{D}_{\text {lung }}\right) \times 100$, where $\mathrm{MaxD}_{\text {mediastinal }}$ and $\max \mathrm{D}_{\text {mediastinal }}$ are the maximum long-axis and short-axis diameter on mediastinal window, respectively; $\mathrm{MaxD}_{\text {lung }}$ and max $D_{\text {lung }}$ are the maximum long-axis and short-axis diameter on lung window, respectively (Figure 1 shows examples of measurement).
Table 1 The definitions and assignments of CT features for stage I lung adenocarcinoma

\begin{tabular}{|c|c|}
\hline CT features & Definitions and assignments \\
\hline \multicolumn{2}{|l|}{ Size } \\
\hline Long-axis diameter & $\begin{array}{l}\text { Maximum diameter in axial section on } \\
\text { lung window }\end{array}$ \\
\hline Short-axis diameter & $\begin{array}{l}\text { Maximum perpendicular diameter in the } \\
\text { same section on lung window }\end{array}$ \\
\hline \multicolumn{2}{|l|}{ Appearance } \\
\hline Shape & $\begin{array}{l}\text { The overall shape of tumor by visual } \\
\text { inspection }\end{array}$ \\
\hline Round & 1 \\
\hline Oval & 2 \\
\hline Irregular & 3 \\
\hline Lobulation & $\begin{array}{l}\text { Arc-shape or wavelike structure on the } \\
\text { surface of tumor }\end{array}$ \\
\hline None & 1 \\
\hline Number $<3$ & 2 \\
\hline Number $\geq 3$ & 3 \\
\hline \multicolumn{2}{|l|}{ Margin } \\
\hline Border & Clear or obscure boundary of tumor \\
\hline Clear & 1 \\
\hline Somewhat obscure & 2 \\
\hline Obscure & 3 \\
\hline Spiculation & $\begin{array}{l}\text { The length of needle-like protuberance } \\
\text { from tumor margin to peripheral lung } \\
\text { parenchyma }\end{array}$ \\
\hline None & 1 \\
\hline Length $<5 \mathrm{~mm}$ & 2 \\
\hline Length $\geq 5 \mathrm{~mm}$ & 3 \\
\hline \multicolumn{2}{|l|}{ Intratumoral } \\
\hline Attenuation types & $\begin{array}{l}\text { Tumors are divided into: GGO, mix- } \\
\text { GGO, and solid in terms of attenuation } \\
\text { on CT }\end{array}$ \\
\hline GGO & 1 \\
\hline Mixed-GGO & 2 \\
\hline Solid & 3 \\
\hline Vacuolation & Bubble-like lucency inside the tumor \\
\hline Absence & 0 \\
\hline Presence & 1 \\
\hline
\end{tabular}

Table 1 (continued) 
Table 1 (continued)

\begin{tabular}{cl}
\hline CT features & Definitions and assignments \\
\hline Air bronchogram & $\begin{array}{l}\text { Columnar air structure in multiple } \\
\text { consecutive sections inside the tumor }\end{array}$ \\
Absence & 1 \\
Presence & 0
\end{tabular}

Bronchial truncation Columnar air structure inside the tumor was sharply obstructed

$\begin{array}{lc}\begin{array}{l}\text { Absence } \\ \text { Presence }\end{array} & 0 \\ \begin{array}{l}\text { Vascular invasion } \\ \text { Intratumor vessels were disrupted } \\ \text { sharply or gradually and embedded } \\ \text { inside the tumor }\end{array} \\ \begin{array}{l}\text { Absence } \\ \text { Presence }\end{array} \\ \begin{array}{l}\text { Veritumoral } \\ \text { Vascular convergence }\end{array} \\ \text { One or more vessels were pulled and } \\ \text { converged towards tumor }\end{array}$

No obvious vascular 0

convergence

Obvious vascular
convergence

Thickened

bronchovascular

bundles

Absence
Presence

Fissure attachment Tumor attached to the fissure

$\begin{array}{lr}\text { Absence } & 0 \\ \text { Presence } & 1\end{array}$

Pleural attachment Tumor attached to the pleura

$\begin{array}{ll}\text { Absence } & 0 \\ \text { Presence } & 1\end{array}$

Pleural retraction The pleura was pulled by tumor and formed a cloak-like structure

$\begin{array}{ll}\text { Absence } & 0 \\ \text { Presence } & 1\end{array}$

Peripheral Peripheral emphysema defined as wellemphysema bounded areas of decreased attenuation by visual assessment, including centrilobular, paraseptal, whole lobular or mixed emphysema

Table 1 (continued)
Table 1 (continued)

\begin{tabular}{cc}
\hline CT features & Definitions and assignments \\
\hline Absence & 0 \\
Presence & 1 \\
Peripheral fibrosis & $\begin{array}{l}\text { Peripheral fibrosis included reticular } \\
\text { opacity, honeycombed structure, } \\
\text { tractive bronchiectasis, GGO, } \\
\text { consolidation and subpleural line }\end{array}$ \\
Absence & 0 \\
Presence & 1 \\
\hline GGO, ground-glass opacity.
\end{tabular}

\section{Ki-67 LI and serum tumor markers detection}

The Ki-67 expression of tumor samples from surgical resection was routinely detected with the $\mathrm{Ki}-67$ protein antibody (Maixin Biotechnology Corporation, Fuzhou, China) at a dilution of 1:50 via immunohistochemistry. Five fields of view under high magnification were selected from every section to count positive cells, the whole or partial nuclei of which were stained to be brown. The percentage of positive cells in 1,000 tumor cells was calculated. Two categories were defined according to the percentage of $\mathrm{Ki}$ 67 positive cells: low Ki-67 expression $(<10 \%)$ and high Ki67 expression ( $\geq 10 \%)$ in accordance with a recent study (11). To explore the association between Ki-67 expression distribution and histological subtypes, we further divided samples into four Ki-67 subgroups: $<10 \%, 10-25 \%$, $25-50 \%$, and $\geq 50 \%$.

Serum CEA and CYFRA21-1 were detected with enzyme-linked immunosorbent assay. We regarded 5 and $3.3 \mu \mathrm{g} / \mathrm{L}$ as the cutoff values for CEA and CYFRA21-1, respectively, which accorded with instructions of the assay kits. TMI was calculated with the following formula: TMI = square root of $[($ serum CEA concentration $/ 5.0 \mu \mathrm{g} / \mathrm{L}) \times$ (serum CYFRA 21-1 concentration/3.3 $\mathrm{g} / \mathrm{L}$ )] (15). We divided patients into two groups: TMI $\leq 1.0$ and TMI $>1.0$ by taking 1.0 as the cutoff, which was obtained when the concentrations of CEA and CYFRA 21-1 were equal to cutoff values.

\section{Statistical analysis}

All statistical analysis was conducted with SPSS (IBM, ver. 26.0) statistics software. The intraclass correlation coefficient was computed for continuous variables. The 

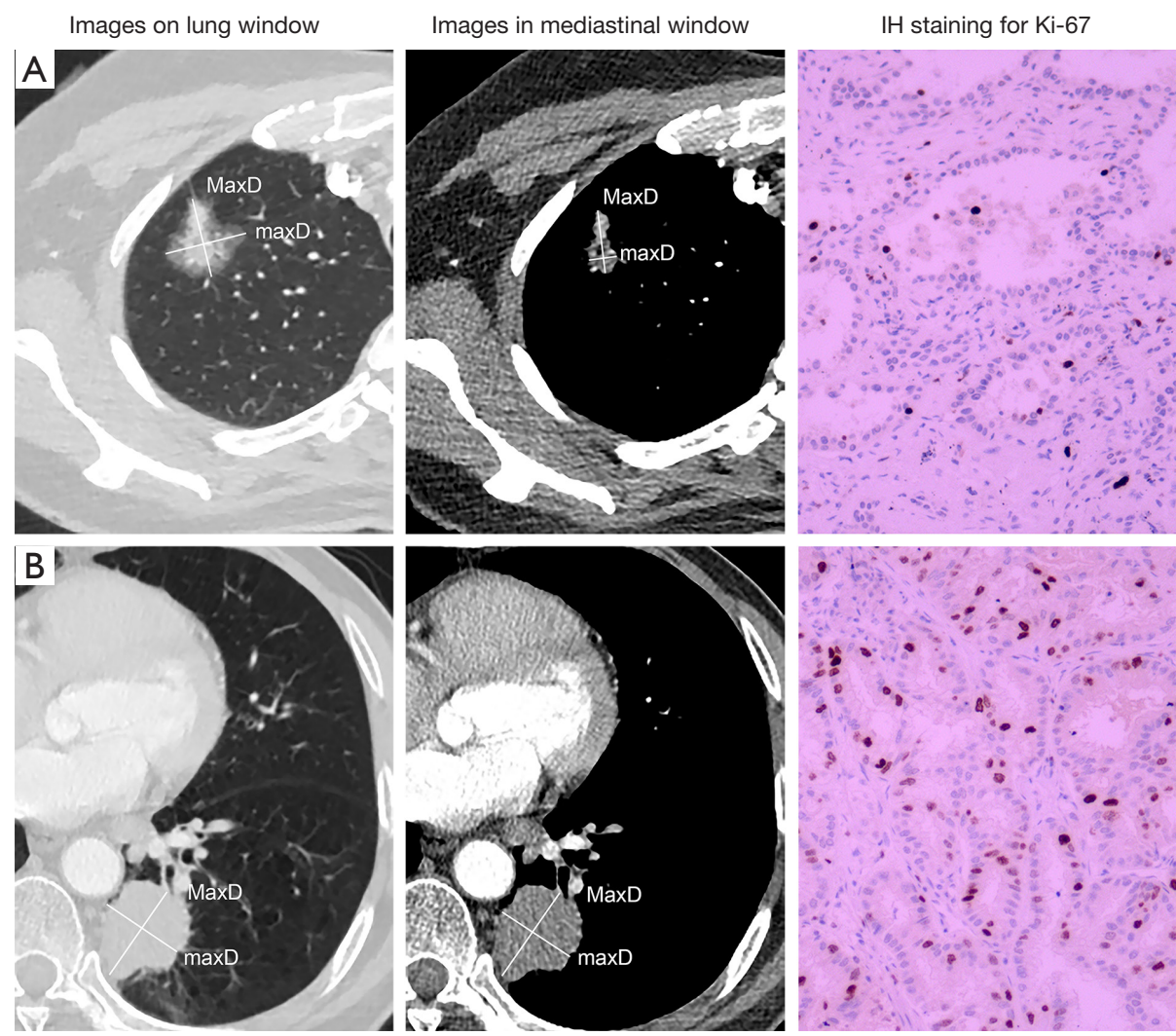

Figure 1 CT images and IH staining images for Ki-67 in the Ki-67 low-expresser and high-expresser. CT images and IH staining (magnification $\times 100$ ) images for Ki-67 in a 46-year-old woman with low Ki-67 expression (A) and a 71-year-old man with high Ki-67 expression (B). The MaxD and maxD on lung window and on mediastinal window were respectively measured to calculate TDR. TDR was $72.92 \%$ and $8.57 \%$ in (A) and (B), respectively. IH, immunohistochemical; MaxD, maximum long-axis diameter; maxD, maximum short-axis diameter; TDR, tumor shadow disappearance rate.

interobserver agreement was evaluated with weighted Kappa coefficient and Cohen's Kappa coefficient for ordered and unordered categorical variables, respectively. The differences of continuous variables were compared by Mann-Whitney U test or Kruskal-Wallis test. The categorical variables described as frequency were compared using Chi-square test or Fisher's exact test. Pearson correlation analysis was used to assess the correlation between Ki-67 LI and TDR. The binary logistic regression analysis was employed to identify independent factors associated with Ki-67 levels. $\mathrm{P}<0.05$ indicated a statistically significant difference.

\section{Results}

In total, 182 cases (medium age, 59 years; age range, 33-78 years) of stage I ADC were enrolled in the study. Of them, 107
(58.8\%) patients were male (medium age, 60 years; range, $37-78$ years) and 75 (41.2\%) patients were female (medium age, 57 years; age range, 33-75 years). There were 73 (40.1\%) Ki-67 high-expressers and 109 (59.9\%) lowexpressers out of 182 patients.

\section{Interobserver agreement assessment}

The interobserver agreement of CT features between two radiologists was good as detailed in Table S1. The intraclass correlation coefficients for TDR, long-axis diameter and shortaxis diameter was 0.970 (range, 0.960-0.978), 0.994 (range, 0.991-0.996) and 0.994 (range, 0.992-0.996), respectively.

\section{Clinicopathological characteristics and Ki-67 expression}

As showed in Table 2, Ki-67 high-expressers were more 
Table 2 The associations among clinicopathological characteristics, serum tumor markers, and Ki-67 expression levels

\begin{tabular}{|c|c|c|c|c|}
\hline Variables & Total & \multicolumn{2}{|r|}{ Ki-67 } & $\mathrm{P}^{\star}$ value \\
\hline $\mathrm{Age}^{\ddagger}$ (year) & $59(52,63)$ & $58(52,63)$ & $59(52,63)$ & 0.742 \\
\hline Gender & & & & 0.001 \\
\hline Female & 75 & 41 & 34 & \\
\hline Smoking history & & & & 0.027 \\
\hline Non-smoker & 108 & 52 & 56 & \\
\hline Ex-smoker & 30 & 9 & 21 & \\
\hline Current smoker & 44 & 12 & 32 & \\
\hline Well-differentiated & 38 & 27 & 11 & \\
\hline Moderately-differentiated & 77 & 34 & 43 & \\
\hline Poorly-differentiated & 64 & 10 & 54 & \\
\hline Unknown & 3 & 2 & 1 & \\
\hline TNM stage & & & & 0.022 \\
\hline IA & 130 & 59 & 71 & \\
\hline IB & 52 & 14 & 38 & \\
\hline Lobe location & & & & 0.804 \\
\hline Serum CEA status (ug/L) & & & & 0.002 \\
\hline$\leq 5$ & 135 & 63 & 72 & \\
\hline$>5$ & 47 & 10 & 37 & \\
\hline Serum CYFRA 21-1 status (ug/L) & & & & 0.040 \\
\hline$\leq 3.3$ & 149 & 65 & 84 & \\
\hline$>3.3$ & 33 & 8 & 25 & \\
\hline TMI status & & & & 0.001 \\
\hline$\leq 1$ & 146 & 67 & 79 & \\
\hline$>1$ & 36 & 6 & 30 & \\
\hline
\end{tabular}

\footnotetext{
${ }^{\dagger}$, histological grade was determined according to the new grading system endorsed by IASLC pathology committee in 2020; ${ }^{\ddagger}$, data were presented as medium (inter-quartiles). Ex-smokers are defined as those who have smoked cigarettes of $\geq 100$ in their lifetime but have quit at the time of preoperative CT examination; *, Mann-Whitney $U$ test was used for continuous data and Chi-square test was used for categorical data. CEA, carcinoembryonic antigen; CYFRA 21-1, cytokeratin 19 fragments; TMI, tumor maker index.
} 
frequent in males than females $(\mathrm{P}=0.001)$, patients with smoking history $(\mathrm{P}=0.027)$, higher pack-year $(\mathrm{P}=0.010)$, worse differentiation $(\mathrm{P}<0.001)$, stage IB than IA $(\mathrm{P}=0.022)$, CEA $>5 \mu \mathrm{g} / \mathrm{L}(\mathrm{P}=0.002)$, CYFRA $21-1>3.3 \mu \mathrm{g} / \mathrm{L}(\mathrm{P}=0.040)$, and TMI $>1(\mathrm{P}=0.001)$. Serum CEA and TMI values in Ki-67 high-expressers were significantly higher than those in Ki-67 low-expressers ( $\mathrm{P}=0.007$ and 0.002 , respectively); CYFRA 21-1 values had no significant difference between Ki67 low-expressers and high-expressers $(\mathrm{P}=0.097)$ (Figure 2).

As revealed in Table 3 and Figure 3A, SPA [60 (40, 60)] had the highest Ki-67 LI, followed by MPA [30 (10, 40)], PPA $[10(8,20)]$, and APA $[10(5,27.5)]$, while LPA [5 $(3,10)]$ had the lowest Ki-67 LI $(\mathrm{P}<0.001)$. The Ki-67 subgroup of $<10 \%$ was composed of APA $(40.9 \%)$, LPA (39.4\%) and PPA (19.7\%) with no MPA and SPA, while the Ki-67 subgroup of $\geq 50 \%$ mainly consisted of SPA (65\%) and APA (25\%); APA and PPA were the primary histological subtypes both in $\mathrm{Ki}-67$ subgroups of $10-25 \%$ and $25-50 \%$ $(\mathrm{P}<0.001$; Table 3 and Figure 3B). Accordingly, Ki-67 LI in $\mathrm{ADC}$ with micropapillary and/or solid components [30 $(10,50)]$ was significantly higher than that in $\mathrm{ADC}$ without micropapillary and/or solid components $[8(5,15), \mathrm{P}<0.001$; Figure 3C].

\section{CT features and Ki-67 expression}

As Table 4 showed, TDR in Ki-67 high-expressers (Figure 1B) was significantly lower than in Ki-67 low-expressers (Figure $1 A)(\mathrm{P}<0.001)$. Furthermore, TDR had a negative correlation with Ki-67 LI ( $\mathrm{r}=-0.478, \mathrm{P}<0.001$; Figure 4). The long-axis diameter $(\mathrm{P}=0.020)$ and short-axis diameter $(\mathrm{P}=0.018)$ in Ki-67 high-expressers were significantly greater than those in Ki-67 low-expressers. Tumors with more lobules $(\mathrm{P}=0.006)$ and spicules $(\mathrm{P}<0.001)$, vacuolation $(\mathrm{P}=0.032)$, vascular invasion $(\mathrm{P}<0.001)$, vascular convergence $(\mathrm{P}<0.001)$, thickened bronchovascular bundles $(\mathrm{P}=0.043)$, pleural attachment $(\mathrm{P}=0.032)$, and peripheral fibrosis $(\mathrm{P}=0.040)$, and solid tumors $(\mathrm{P}<0.001)$ were more frequently observed in Ki-67 high-expressers.

\section{Multivariable analysis of factors associated with Ki-67 levels}

All statistically significant variables in univariate analysis were included into binary logistic regression analysis. As Table 5 showed, gender [odds ratio (OR): 2.87; 95\% confidence interval (CI): 1.27, 6.45], histological grade (OR of poorly-differentiated: $3.81,95 \%$ CI: $1.38,10.53$; OR of moderately-differentiated: $1.33,95 \%$ CI: $0.55,3.21$ ), TDR (OR: 0.97, 95\% CI: 0.96, 0.98) and attenuation types (OR: 4.72, 95\% CI: 2.15, 10.38) were independent factors associated with Ki-67 expression levels.

\section{Discussion}

In this study, we evaluated Ki-67 expression among different ADC histological subtypes and found that SPA had the highest Ki-67 LI, followed by MPA, PPA and APA, while LPA had the lowest Ki-67 LI. Multivariate regression analysis revealed that gender, histological grade, TDR and tumor attenuation types on CT were independently associated with $\mathrm{Ki}-67$ expression levels in stage I ADC. To our best knowledge, this is the first study to expound the relationships among histological grade, TMI, morphological CT features, and Ki-67 expression in stage I ADC.

Although the exact biological mechanism of Ki-67 protein acting on cell proliferation is uncertain, it was found that Ki-67 played a role in the transcription process of ribosomal RNA and Ki-67 protein inactivation inhibited the synthesis of ribosomal RNA (19). It is no doubt that $\mathrm{Ki}-67 \mathrm{LI}$, as a reflection of cell proliferation activity, tends to increase with invasion development in malignant carcinoma. TDR is a new radiological indicator derived from the ratio of tumor's maximum diameters on mediastinal window to those on lung window manually-measured on CT images, which actually reveals the percentile of solid component on tumor's maximum section. The lower TDR signifies a greater proportion of solid components contained in tumor. In addition to this relatively objective measure, we classified tumors into three categories: ground-glass opacity (GGO), mixed-GGO and solid nodules by subjective interpretation of CT attenuation. As reported by Okada et al. (20), lower TDR and GGO proportion on CT were linked with stronger tumor aggressiveness both in lymphatic and vascular structures. This result was also supported by Hattori et al. who demonstrated the solid component is a well-established marker for invasiveness (18). The present study revealed that solid nodule and lower TDR were more frequently observed in Ki-67 high-expressers than in lowexpressers. Furthermore, TDR was negatively correlated with Ki-67 LI with Pearson correlation coefficient of -0.478 , indicating the more solid component in tumor, the higher Ki-67 LI can be observed. Considering high $\mathrm{Ki}-67 \mathrm{LI}$ is a hallmark of aggressiveness, this conclusion was consistent with the findings of Okada et al. and Hattori et al. The routine approach to detect $\mathrm{Ki}-67 \mathrm{LI}$ is 

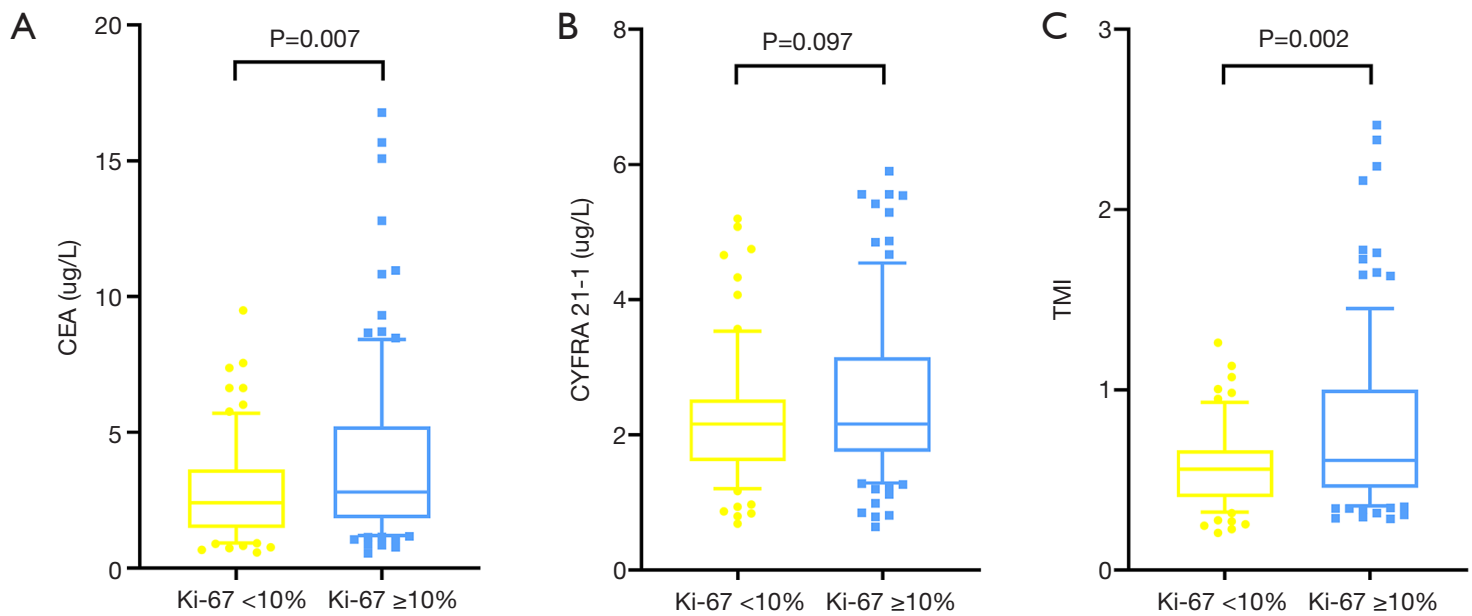

Figure 2 Serum CEA, CYFRA 21-1, and TMI values according to Ki-67 expression. Boxplots and dotplots graphically depict the distributions of serum CEA (A), CYFRA 21-1 (B), and TMI (C) values in Ki-67 low-expressers and Ki-67 high-expressers. CEA, carcinoembryonic antigen; TMI, tumor maker index.

Table 3 The Ki-67 expression in different histological subtypes of lung adenocarcinoma

\begin{tabular}{|c|c|c|c|c|c|c|}
\hline Variables & \multicolumn{5}{|c|}{ ADC histological subtypes } & $P$ value \\
\hline $\mathrm{Ki}-67^{\ddagger}(\%)$ & $5(3,10)$ & $10(5,27.5)$ & $10(8,20)$ & $30(10,40)$ & $60(40,60)$ & $<0.001$ \\
\hline Ki-67 subgroups & & & & & & $<0.001$ \\
\hline$<10 \%$ & 28 & 29 & 14 & 0 & 0 & \\
\hline $25-50 \%$ & 3 & 15 & 6 & 2 & 4 & \\
\hline$\geq 50 \%$ & 0 & 5 & 1 & 1 & 13 & \\
\hline
\end{tabular}

${ }^{\ddagger}$, Data were presented as medium (inter-quartiles). ADC, adenocarcinoma; LPA, lepidic predominant adenocarcinoma; APA, acinar predominant adenocarcinoma; PPA, papillary predominant adenocarcinoma; MPA, micropapillary predominant adenocarcinoma; SPA, solid predominant adenocarcinoma.
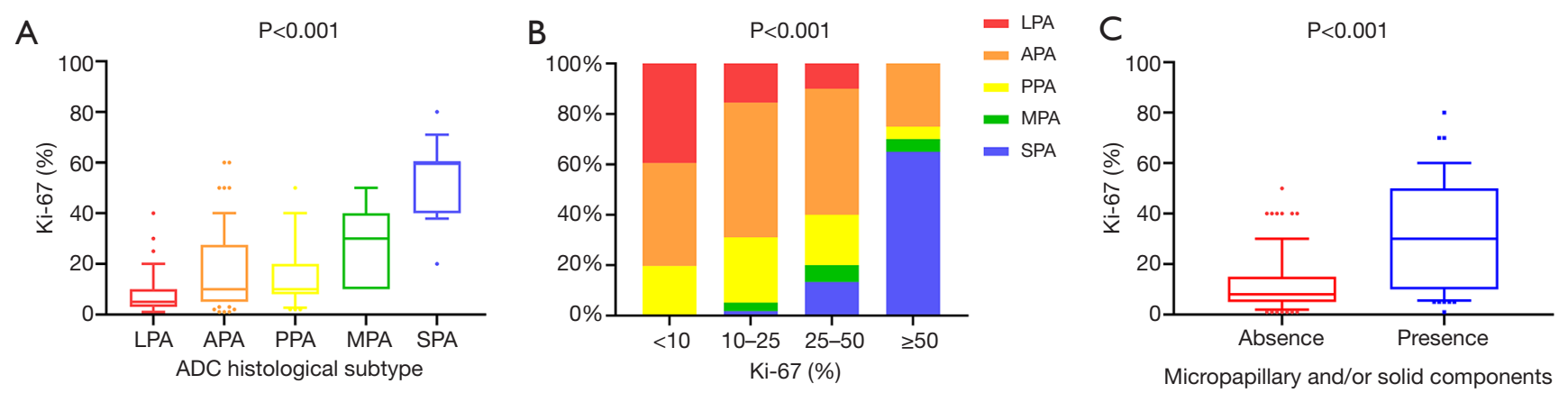

Figure $3 \mathrm{Ki}-67$ expression according to histological subtypes of lung adenocarcinoma. The distribution of Ki-67 expression in terms of different histological subtypes of lung adenocarcinoma (A,B) and Ki-67 expression between tumors with and without micropapillary and/or solid components (C). LPA, lepidic predominant adenocarcinoma; APA, acinar predominant adenocarcinoma; PPA, papillary predominant adenocarcinoma; MPA, micropapillary predominant adenocarcinoma; SPA, solid predominant adenocarcinoma. 
Table 4 The association between CT features and Ki-67 expression levels

\begin{tabular}{|c|c|c|c|c|}
\hline Variables & Total & \multicolumn{2}{|c|}{ Ki-67 } & $\mathrm{P}^{*}$ value \\
\hline Long-axis diameter ${ }^{\ddagger}(\mathrm{mm})$ & $25.0(19.5,32.5)$ & $23.5(17.0,29.3)$ & $26.5(20.3,34.5)$ & 0.020 \\
\hline Short-axis diameter ${ }^{\ddagger}(\mathrm{mm})$ & $20.5(15.0,26.0)$ & $18.0(13.5,24.5)$ & $21.0(16.3,27.3)$ & 0.018 \\
\hline $\operatorname{TDR}^{\ddagger}(100 \%)$ & $38.4(24.3,79.5)$ & $79.2(49.5,97.8)$ & $30.0(19.9,43.3)$ & $<0.001$ \\
\hline 1 & 44 & 22 & 22 & \\
\hline 2 & 104 & 38 & 66 & \\
\hline 3 & 34 & 13 & 21 & \\
\hline Lobulation & & & & 0.006 \\
\hline 3 & 114 & 36 & 78 & \\
\hline Border & & & & 0.847 \\
\hline 1 & 5 & 3 & 2 & \\
\hline 2 & 32 & 12 & 20 & \\
\hline 3 & 145 & 58 & 87 & \\
\hline Spiculation & & & & $<0.001$ \\
\hline 1 & 63 & 40 & 23 & \\
\hline 2 & 71 & 20 & 51 & \\
\hline 0 & 134 & 60 & 74 & \\
\hline 1 & 48 & 13 & 35 & \\
\hline Air bronchogram & & & & 0.344 \\
\hline 0 & 75 & 27 & 48 & \\
\hline 1 & 107 & 46 & 61 & \\
\hline Bronchial truncation & & & & 0.324 \\
\hline 0 & 122 & 52 & 70 & \\
\hline 1 & 60 & 21 & 39 & \\
\hline Vascular invasion & & & & $<0.001$ \\
\hline 0 & 97 & 57 & 40 & \\
\hline 1 & 85 & 16 & 69 & \\
\hline
\end{tabular}

Table 4 (continued) 
Table 4 (continued)

\begin{tabular}{|c|c|c|c|c|}
\hline Variables & Total & \multicolumn{2}{|c|}{$\mathrm{Ki}-67$} & $\mathrm{P}^{*}$ value \\
\hline Vascular convergence & & & & $<0.001$ \\
\hline 0 & 48 & 32 & 16 & \\
\hline 1 & 134 & 41 & 93 & \\
\hline 0 & 135 & 60 & 75 & \\
\hline 1 & 47 & 13 & 34 & \\
\hline Fissure attachment & & & & 0.769 \\
\hline 0 & 135 & 55 & 80 & \\
\hline 0 & 134 & 60 & 74 & \\
\hline 1 & 48 & 13 & 35 & \\
\hline Pleural retraction & & & & 0.140 \\
\hline 0 & 33 & 17 & 16 & \\
\hline 1 & 149 & 56 & 93 & \\
\hline Peripheral emphysema & & & & 0.053 \\
\hline 0 & 147 & 64 & 83 & \\
\hline 1 & 35 & 9 & 26 & \\
\hline
\end{tabular}

${ }^{\ddagger}$, data were presented as medium (inter-quartiles); *, Mann-Whitney $U$ test was used for continuous data and Chi-square test was used for categorical data. TDR, tumor shadow disappearance rate.

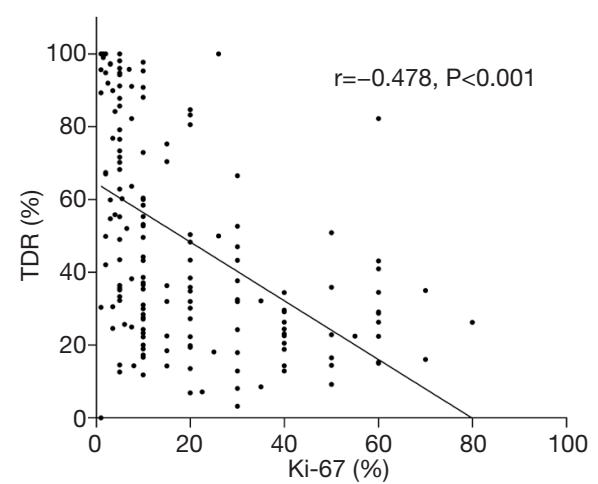

Figure 4 Scatter diagram and linear regression line show a negative correlation between TDR and Ki-67 ( $\mathrm{r}=-0.478, \mathrm{P}<0.001)$. TDR, tumor shadow disappearance rate. immunohistochemistry through sampling from histological specimens acquired from biopsy or surgical resection. Nevertheless, the potential risk in misdiagnosis of Ki-67 LI on account of sampling errors remain limitations of the method. In regard to the associations of TDR and tumor attenuation with $\mathrm{Ki}-67$, we inferred that the solid area of tumor on CT has higher Ki-67 LI, whereas the GGO area has relatively lower Ki-67 LI. This information will hopefully direct and assist pathologists in more accurately sampling from tumor tissue and thereby in avoiding underestimation of $\mathrm{Ki}-67$ expression.

It is worth noting that histological grade was another independent factor associated with $\mathrm{Ki}-67$ expression. In this study, the new grading system proposed by IASLC 
Table 5 The independent factors associated with Ki-67 expression levels

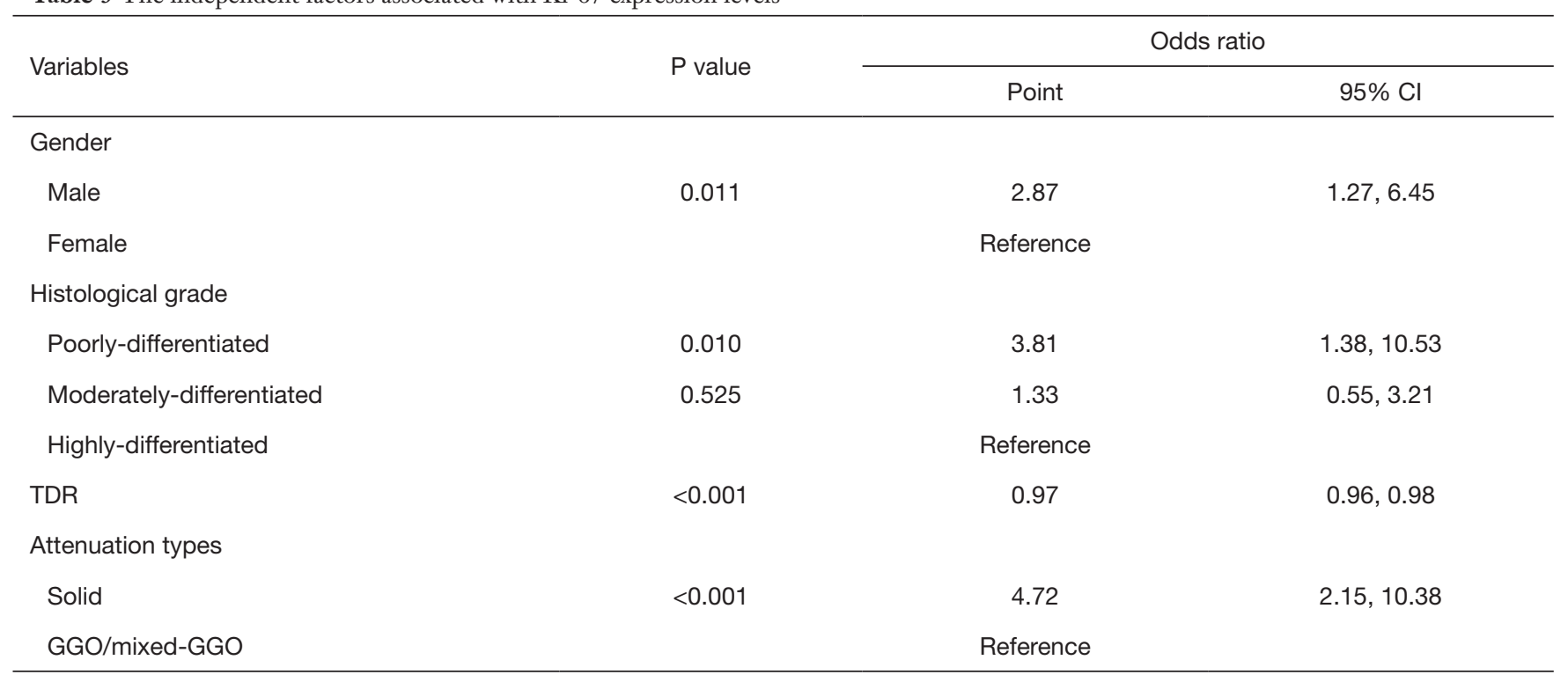

95\% Cl, 95\% confidence interval; TDR, tumor shadow disappearance rate; GGO, ground-glass opacity.

pathology committee was adopted to determine the histological grade, which is based on the predominant architecture and the proportion of high-grade histological patterns instead of the predominant pattern only (6). The IASLC grading system considered the histological heterogeneity and served as a strong prognostic classifier of invasive ADC. Surprisingly, we also found that Ki-67 expression differed significantly among ADC histological subtypes with an increasing trend across LPA, APA, PPA, MPA and SPA. Furthermore, tumors with micropapillary and/or solid components had higher Ki-67 expression than those without micropapillary and/or solid components. It was figured out whether differential expressions of $\mathrm{Ki}$ 67 across ADC histological subtypes could contribute to the known prognostic differences among them. As we expected, a recent literature indicated that the survival difference between LPA/APA/PPA and MPA/ SPA became insignificant when Ki-67 expression was comparable (11), which was in favor of our assumption. More well-designed studies are warranted to verify these observations.

Serum CEA and TMI values in Ki-67 high-expressers were significantly higher than those in Ki-67 lowexpressers, and Ki-67 high-expressers were more prone to harbor serum CYFRA 21-1 >3.3 $\mu \mathrm{g} / \mathrm{L}$ in univariate analysis. Reasonably, the novel variable TMI combining influences of both serum CEA and CYFRA 21-1 also revealed a significant difference between Ki-67 high-expressers and low-expressers. Previous studies reported that elevated serum CEA in tumor patients may attribute to locally pleural and vascular invasion by malignant cells and serum CYFRA 21-1 is mainly correlated with tumor burden $(13,14)$. Therefore, it can be deduced that higher Ki-67 expression indicating more active tumor cell proliferation and more aggressive behaviors in tumor is closely associated with increased serum CYFRA 21-1 and CEA level.

Apart from that, this study showed that significantly high $\mathrm{Ki}-67$ expression was more frequent in males than that in females, in accordance with previous literature (21-23). We also found smokers, especially the currentsmokers and with larger pack-year were more likely to be Ki-67 high-expressers. These results lead to speculation on whether males with a predisposition in smoking will contribute to high Ki-67 expression, but it should be confirmed by future in-depth research. Among other CT features, we found that tumors with larger diameters, more lobules and spicules, vacuolation, vascular invasion, vascular convergence, thickened bronchovascular bundles, pleural attachment, and peripheral fibrosis significantly had higher Ki-67 expression. Prior to this study, there were limited available studies reporting the relationship between $\mathrm{Ki}$ 67 and CT morphological features. Gu et al. found that signs of lobulation and spiculation, pleural indentation have strengths to discriminate between high and low Ki67 expression groups in 245 patients with NSCLC (21). Yan et al. examined 95 resected ADCs and found that the diameter of tumor, density from GGO to mixed GGO to 
solid nodules, rate of lobulation signs gradually increased with Ki-67 expression level, while Ki-67 expression had no significant correlation with spiculation and pleural retraction (24). Some of these findings are consistent with our results $(21,24)$, but some are conflicting (24). Contributing to the discrepancy may be differences in inclusion criteria and Ki-67 cut-off values.

As we all know, TNM stage is the most important factor of prognostic value in tumor treatment (25). Tumors in different TNM stages appear diverse performances of aggressiveness, which can manifest as distinct morphological signs in CT and Ki-67 expression. Meanwhile, variations of histological subtypes of NSCLC are in biologic characters, therapeutic methods and outcomes, and might also reflect in molecular expression including Ki-67. Therefore, different from past studies, we exclusively included patients with stage I ADC, and thus avoided potential confounding factors caused by changes in pathological stage and subtype of lung cancer.

Stage I ADC is substantially recommended to perform surgical resection but have wide variations in outcomes $(25,26)$. It is of vital importance to identify stage I adenocarcinoma patients with poor prognosis, who could benefit from more individualized treatment and prolong their survival. In this study, male patients with worse differentiation, lower TDR and solid tumors were prone to be Ki-67 high-expressers. Theoretically, the factors independently associated with high $\mathrm{Ki}-67$ expression might also have adverse impacts on prognosis in stage I ADC. It is expected that a combination of Ki-67 and these factors would more accurately predict prognosis and stratify patients with resected stage I ADC. Further, a prospective, large-sample, survival research in stage I ADC is necessary to affirm the prognostic value of the independent factors in this study.

There were several limitations in this study. First, due to our retrospective study and including only one research institution, there were inevitable selection biases in this study. Second, we only divided tumors into three categories in terms of CT attenuation, failed to quantify solid components in mixed-GGO, which is a welldocumented metric that also correlates with prognosis. It's needed to complement this metric further. Third, this study exclusively focused on exploring relationships among histological grade, TMI, CT features, and Ki-67 expression, so no survival analysis was conducted. Future work should be conducted to make up for the drawbacks in sample size, quantification of solid components, and survival analysis.

In conclusion, gender, histological grade, TDR and tumor attenuation based on CT were independently associated with Ki-67 expression in stage I ADC. Ki-67 expression differed distinctly according to ADC histological subtypes. Further, we would excavate the incremental value of these independent factors with respect to Ki-67 and the prognostic role of Ki-67 across different ADC histological subtypes in survival analysis.

\section{Acknowledgments}

We would like to acknowledge Zujun Hou for the comments on this manuscript. We would like to acknowledge Wei Wu for the language revision of this manuscript.

Funding: This study was supported by the National Natural Science Foundation of China (NO. 81873889).

\section{Footnote}

Reporting checklist: The authors have completed the MDAR checklist. Available at http://dx.doi.org/10.21037/jtd-21-7

Data Sharing Statement: Available at http://dx.doi. org/10.21037/jtd-21-7

Peer Review File: Available at http://dx.doi.org/10.21037/jtd21-7

Conflicts of Interest: All authors have completed the ICMJE uniform disclosure form (available at http://dx.doi. org/10.21037/jtd-21-7). The authors have no conflicts of interest to declare.

Ethical Statement: The authors are accountable for all aspects of the work in ensuring that questions related to the accuracy or integrity of any part of the work are appropriately investigated and resolved. The study was conducted in accordance with the Declaration of Helsinki (as revised in 2013). This study was approved by the Institutional Review Board (NO. TJ-IRB-20180615). Individual consent for this retrospective analysis was waived.

Open Access Statement: This is an Open Access article distributed in accordance with the Creative Commons Attribution-NonCommercial-NoDerivs 4.0 International License (CC BY-NC-ND 4.0), which permits the noncommercial replication and distribution of the article with the strict proviso that no changes or edits are made and the original work is properly cited (including links to both the formal publication through the relevant DOI and the license). 
See: https://creativecommons.org/licenses/by-nc-nd/4.0/.

\section{References}

1. Li P, Gao Q, Jiang X, et al. Comparison of Clinicopathological Features and Prognosis between ALK Rearrangements and EGFR Mutations in Surgically Resected Early-stage Lung Adenocarcinoma. J Cancer 2019;10:61-71.

2. $\mathrm{Xu} \mathrm{J,} \mathrm{Liu} \mathrm{P,} \mathrm{Da} \mathrm{J,} \mathrm{et} \mathrm{al.} \mathrm{Prognostic} \mathrm{value} \mathrm{of} \mathrm{Ki-67} \mathrm{in} \mathrm{stage} \mathrm{I}$ non-small-cell lung cancer: A meta-analysis involving 1931 patients. Pathol Res Pract 2019;215:855-60.

3. Dai J, Liu M, Yang Y, et al. Optimal Lymph Node Examination and Adjuvant Chemotherapy for Stage I Lung Cancer. J Thorac Oncol 2019;14:1277-85.

4. Horn L, Sandler AB, Putnam JB, et al. The rationale for adjuvant chemotherapy in stage I non-small cell lung cancer. J Thorac Oncol 2007;2:377-83.

5. Travis WD, Brambilla E, Noguchi M, et al. International association for the study of lung cancer/american thoracic society/european respiratory society international multidisciplinary classification of lung adenocarcinoma. J Thorac Oncol 2011;6:244-85.

6. Moreira AL, Ocampo PSS, Xia Y, et al. A Grading System for Invasive Pulmonary Adenocarcinoma: A Proposal From the International Association for the Study of Lung Cancer Pathology Committee. J Thorac Oncol 2020;15:1599-610.

7. Pan DH, Wen DY, Luo YH, et al. The diagnostic and prognostic values of Ki-67/MIB-1 expression in thyroid cancer: a meta-analysis with 6,051 cases. Onco Targets Ther 2017;10:3261-76.

8. Ács B, Zámbó V, Vízkeleti L, et al. Ki-67 as a controversial predictive and prognostic marker in breast cancer patients treated with neoadjuvant chemotherapy. Diagn Pathol 2017;12:20.

9. Wei DM, Chen WJ, Meng RM, et al. Augmented expression of $\mathrm{Ki}-67$ is correlated with clinicopathological characteristics and prognosis for lung cancer patients: an up-dated systematic review and meta-analysis with 108 studies and 14,732 patients. Respir Res 2018;19:150.

10. Maki Y, Soh J, Ichimura K, et al. Impact of GLUT1 and Ki-67 expression on early-stage lung adenocarcinoma diagnosed according to a new international multidisciplinary classification. Oncol Rep 2013;29:133-40.

11. Li Z, Li F, Pan C, et al. Tumor cell proliferation (Ki-67) expression and its prognostic significance in histological subtypes of lung adenocarcinoma. Lung Cancer
2021;154:69-75.

12. Warth A, Cortis J, Soltermann A, et al. Tumour cell proliferation (Ki-67) in non-small cell lung cancer: a critical reappraisal of its prognostic role. Br J Cancer 2014;111:1222-9.

13. Matsuguma H, Nakahara R, Igarashi S, et al. Pathologic stage I non-small cell lung cancer with high levels of preoperative serum carcinoembryonic antigen: clinicopathologic characteristics and prognosis. J Thorac Cardiovasc Surg 2008;135:44-9.

14. Park SY, Lee JG, Kim J, et al. Preoperative serum CYFRA 21-1 level as a prognostic factor in surgically treated adenocarcinoma of lung. Lung Cancer 2013;79:156-60.

15. Muley T, Fetz TH, Dienemann H, et al. Tumor volume and tumor marker index based on CYFRA 21-1 and CEA are strong prognostic factors in operated early stage NSCLC. Lung Cancer 2008;60:408-15.

16. Liu Y, Kim J, Qu F, et al. CT Features Associated with Epidermal Growth Factor Receptor Mutation Status in Patients with Lung Adenocarcinoma. Radiology 2016;280:271-80.

17. Zhang Y, Shen Y, Qiang JW, et al. CT features distinguishing pre-invasive from invasive pulmonary adenocarcinomas appearing as ground-glass nodules. Eur Radiol 2016;26:2921-8.

18. Hattori A, S. Hirayama, T. Matsunaga, et al. Distinct Clinicopathologic Characteristics and Prognosis Based on the Presence of Ground Glass Opacity Component in Clinical Stage IA Lung Adenocarcinoma. J Thorac Oncol 2019;14:265-75.

19. Jakobsen JN, Sorensen JB. Clinical impact of ki-67 labeling index in non-small cell lung cancer. Lung Cancer 2013;79:1-7.

20. Okada M, Tauchi S, Iwanaga K, et al. Associations among bronchioloalveolar carcinoma components, positron emission tomographic and computed tomographic findings, and malignant behavior in small lung adenocarcinomas. J Thorac Cardiovasc Surg 2007;133:1448-54.

21. Gu Q, Feng Z, Liang Q, et al. Machine learning-based radiomics strategy for prediction of cell proliferation in non-small cell lung cancer. Eur J Radiol 2019;118:32-37.

22. Ahn HK, Jung M, Ha SY, et al. Clinical significance of Ki67 and p53 expression in curatively resected non-small cell lung cancer. Tumour Biol 2014;35:5735-40.

23. Yamashita S, Moroga T, Tokuishi K, et al. Ki-67 labeling index is associated with recurrence after segmentectomy under video-assisted thoracoscopic surgery in stage I non-small cell lung cancer. Ann Thorac Cardiovasc Surg 
2011;17:341-6.

24. Yan J, Wang H, Zhou H, et al. Correlation between expression of Ki-67 and MSCT signs in different types of lung adenocarcinoma. Medicine (Baltimore) 2020;99:e18678.

25. Minami K, Saito Y, Imamura H, et al. Prognostic

Cite this article as: Ma X, Zhou S, Huang L, Zhao P, Wang Y, Hu Q, Xia L. Assessment of relationships among clinicopathological characteristics, morphological computer tomography features, and tumor cell proliferation in stage I lung adenocarcinoma. J Thorac Dis 2021;13(5):2844-2857. doi: $10.21037 /$ jtd-21-7 significance of p53, Ki-67, VEGF and Glut-1 in resected stage I adenocarcinoma of the lung. Lung Cancer 2002;38:51-7.

26. Woo T, Okudela K, Yazawa T, et al. Prognostic value of KRAS mutations and Ki-67 expression in stage I lung adenocarcinomas. Lung Cancer 2009;65:355-62. 


\section{Supplementary}

Table S1 The interobserver agreement of CT features for stage I lung adenocarcinoma

\begin{tabular}{|c|c|c|}
\hline CT features & Kappa value/ICC & $95 \%$ confidence interval \\
\hline $\mathrm{TDR}^{\S}$ & 0.970 & $0.960,0.978$ \\
\hline Long-axis Diameter $^{\S}$ & 0.994 & $0.991,0.996$ \\
\hline Short-axis Diameter ${ }^{\S}$ & 0.994 & $0.992,0.996$ \\
\hline Shape $^{\dagger}$ & 0.865 & $0.799,0.930$ \\
\hline Lobulation $^{\dagger}$ & 0.605 & $0.491,0.719$ \\
\hline Border $^{\dagger}$ & 0.684 & $0.565,0.802$ \\
\hline Spiculation $^{\dagger}$ & 0.663 & $0.573,0.754$ \\
\hline Texture $^{\dagger}$ & 0.894 & $0.833,0.955$ \\
\hline Vacuolation $^{\ddagger}$ & 0.678 & $0.560,0.796$ \\
\hline Air bronchogram ${ }^{\ddagger}$ & 0.721 & $0.619,0.823$ \\
\hline bronchial truncation ${ }^{\ddagger}$ & 0.975 & $0.942,1.008$ \\
\hline Vascular invasion ${ }^{\ddagger}$ & 0.704 & $0.600,0.808$ \\
\hline Vascular convergence ${ }^{\ddagger}$ & 0.673 & $0.546,0.800$ \\
\hline Thickened bronchovascular bundle ${ }^{\ddagger}$ & 0.777 & $0.669,0.885$ \\
\hline Fissure attachment ${ }^{\ddagger}$ & 0.972 & $0.933,1.011$ \\
\hline Pleural attachment ${ }^{\ddagger}$ & 0.958 & $0.911,1.005$ \\
\hline Pleural retraction ${ }^{\ddagger}$ & 0.837 & $0.727,0.947$ \\
\hline Peripheral emphysema ${ }^{\ddagger}$ & 0.747 & $0.622,0.872$ \\
\hline Peripheral fibrosis ${ }^{\ddagger}$ & 0.918 & $0.840,0.996$ \\
\hline
\end{tabular}

$\S$ Intraclass correlation coefficient (ICC) was calculated for continuous variables. ${ }^{\dagger}$ Weighted Kappa coefficient was calculated for ordered categorical variables. ${ }^{\ddagger}$ Cohen’s kappa coefficient was calculated for unordered categorical variables. 